\title{
Rationale for a clinical classification of lymphedema
}

\author{
V Gasbarro ${ }^{1 *}$, S Michelini ${ }^{2}$, E Tsolaki ${ }^{1}$, M Ricci $^{4}$, C Allegra $^{3}$ \\ From de Senectute: Age and Health Forum \\ Catanzaro, Italy. 5-7 December 2009
}

\section{Background}

From a physical, functional and psychological point of view, lymphedema is a chronic, progressive and debilitating disease. For this reason it requires targeted intervention, an early diagnosis and long term follow-up.

Most of the existing classifications are not practical as they are only partial, taking into consideration just one aspect of the disease and are unsuitable for the re-stadiation of lymphedema. We propose an easily applicable and reproducible method for classifying lymphedema which gathers all the important information on the clinical evolution of the disease including a description of its pathophysiology.

\section{Materials and methods}

Between 2002 and 2007, 175 patients with lymphedema of one or more limbs were assessed.

Particular attention was paid to past medical history (infections, traumas, medication and past surgery) ruling out lymphedemas caused by angiodisplastic syndromes. The physical examination included inspection and palpation of the affected $\operatorname{limb}(\mathrm{s})$. We also focused on a careful examination of the skin of the affected limb, searching for changes in skin texture, pitting, hyperkeratosis, fibrosis, infection and elephantiasis, also searching for the presence of the Stemmers sign. In addition, palpation of the affected limb aided in the assessment of spontaneous or evoked pain while palpation of the lymph nodal station of the affected limb was performed to check for signs of lymphadenopathy.

Ultrasound represents a non invasive examination for the evaluation of a swollen limb.

Lymphoscintigraphy provides information on the function of the lymphatic vessels, contributes to the differential diagnosis among the causes of limb edema and evaluates treatment results.

In order to obtain a lymphedema classification based on the clinical, ethological, anatomical and pathophysiologic information obtained, we attempted to reclassify in the same format as the CEAP for venous disorders. In fact, we utilized the acronym C.E.A.P. by adding the letter L to highlight the "lymphedema" aspect.

\section{Conclusions}

The aim of this classification is to provide a comprehensive and descriptive classification that can be globally adopted. During the experimental phase, the objective data obtained provided an accurate description of the abnormalities and facilitated the follow-up of these patients during this period also allowing the re-stadiation of the disease after a period of treatment or in case of worsening of the disease.

We found that it is characterized by an easy applicability since it is based on objective findings obtained by the instrumental tests and does not face the difficulty of dealing with definitions of clinical items as in the C.E.A. $\mathrm{P}$ classification for the chronic venous insufficiency.

\section{Author details}

${ }^{1}$ Departement of Surgery, Anesthesiological and Radiological Sciences, University of Ferrara, Italy. ${ }^{2} \mathrm{~S}$. Giovanni Battista Hospital, Rome, Italy. ${ }^{3}$ Departemento of Angiology St. Giovanni Addolorata, Rome, Italy. ${ }^{4}$ Umberto I Hospital, Ancona, Italy.

Published: 19 May 2010

doi:10.1186/1471-2318-10-S1-A58

Cite this article as: Gasbarro et al:: Rationale for a clinical classification of lymphedema. BMC Geriatrics 2010 10(Suppl 1):A58. 